\title{
MA THEORIE ACIDOPROTEOLYTIQUE SUR LA MATURATION DES FROMAGES
}

\author{
Par le Prof. Constantino GORINI,
}

Directeur du Laboratoire de Bactériologie à l'Insiitut supérieur d'agricuıture de Milan.

Dans le traité l'Industrie fromagère qui est paru récemment, ses auteurs, mon bon ami M. BEAU et M- Ch. BourgatN [1], au chapitre Historique de l'étude de la maturation des fromages disent que mes travaux ont été faits partiellement en collaboration avec le Laboratoire de Liebefeld, c'est-à-dire avec Freudenreich et ses élèves (OrLA Jensen, etc.)

Puisque cette expression pourrait faire attribuer à César ce qui n'est pas à César, j'estime opportun de saisir l'occasion pour exposer un peu d'histoire de ma théorie acidoprotéolytique sur la maturation des fromages, qui a son origine dans des publications italiennes presque inconnues (1).

Dès 1892-1894 [2], d'abord aux Laboratoires de la Santé publique de Rome et ensuite au Laboratoire d'Hygiène de l'Université de Pavie, j'ai découvert les premières bactéries productrices d'acide et de présure (B. prodigiosus, Proteus mirabilis, B. indicus, Ascobacillus citreus), susceptibles d'attaquer la caséine en milieu acide, et j'ai émis l'idée que ces ferments mixtes acidoprotéolytiques pouvaient intervenir dans la maturation des fromages. Il faut se rappeler qu'on était alors à l'aube de la microbiologie fromagère et que deux théories étaient en lutte sur cette maturation: la théorie des ferments peptonisants purs ou Tyrothrix de Duclaux et la théorie des ferments lactiques purs de FreUdenReich ; aucune des deux théories n'était satisfaisante et suffisante, parce que ni les bactéries de Duclaux ni les bactéries de FreuDENREICH n'étaient capables de solubiliser la caséine en milieu acide qui domine et qui doit dominer dans la pâte du fromage de manière à donner des produits de digestion et (non pas de putréfaction) qui caractérisent la maturation normale.

Mon idée rencontra tout de suite un accueil favorable auprès de DUCLAUX, mais non pas auprès de FREUDENRETCH, qui objectait que mes ferments mixtes n'existaient point normalement dans la microflore du lait et du fromage. A vrai dire l'un d'eux, l'Ascobacillus citreus

(1) A ce propos je dois regretter que malheureusement la majorite des auteurs étrangers tombent dans l'inexactitude de ne pas eiter mes travaux publiés en langue italienne, mais seulemeư ceux qui ont été traduits sur des Revues étrangères, où naturellement ils ont paru avee une date postérieure à l'original. C'est ainsi, par exemple, que la méthode de l'examen du fromage par des coupes microscopiques que j'ai préconisée en 1903 (Rend. R. Ist. Lomb. Sc. e Lett., 27 décembre 1903) est souvent datée, par erreur, de l'année 1904, me faisant perdre ainsi ma priorité. 
(qui, aujourd'hui vraisemblablement, est assimilable, aux communs coccus du lait) je l'avais isolé du lait du marché ; mais, ayant dû, à cause de ma carrière, interrompre mes études laitières, je n'ai pas insisté sur l'argument; cependant en 1897, dans un travail [3] concernant l'influence de la température et de l'aérobiose sur les fonctions saccharolytiques et protéolytiques des bactéries, j'ai démontré que pour étudier l'attaque de la caséine par les bactéries, il faut les tenir à des températures basses (1). J'ai soumis mon travail à FREUDENREICH, qui a eu la bonté de le traduire en français pour les Annales de Micrographie [4] qu'il publiait avec Mrques; mais il ne donna aucun signe de modifier son opinion.

En attendant, en Amérique, on avançait une nouvelle théorie qui attribuait la maturation à la diastase protéolytique que BABCocK et Russell avaient rencontrée en 1897 dans le lait, en la considérant comme un produit glandulaire naturel sous le nom de galactase.

En 1901, aussitôt que je fus appelé à l'Ecole supérieure d'agriculture de Milan à créer le premier enseignement de bactériologie agricole en Italie, j'ai voulu aller au Laboratoire de Liebefeld dans le but de vérifier si réellement parmi les bactéries du fromage n'existaient pas des ferments mixtes acidoprotéolytiques.

Dans ce laboratoire, où j'ai demeuré seulement pendant les deux mois de juillet-aout 1901, en appliquant mes méthodes de recherches, j'ai pu démontrer à Freudenreich et OrLa Jensen :

10 $\mathrm{Qu}$ 'en effet aucune des bactéries, auxquelles ils attribuaient exclusivement la maturation du fromage, n'appartenait à la catégorie des ferments mixtes;

$2^{\circ}$ Que pourtant à cette catégorie appartenait un microcoque liquéfiant la gélatine qu'ils avaient bien rencontré dans le fromage, mais n'en ayant pas aperçu le biochimisme particulier sur le lait, ils l'avaient négligé en le considérant comme un microbe banal, inutile, une impureté destinée à être heureusement étouffée par les ferments lactiques purs ;

$3^{\circ}$ Qu'à cette catégorie appartenait aussi un coccus analogue que je venais de découvrir existant normalement dans le lait trait aseptiquement des vaches de l'étable du liebefeld et de deux étables privées voisines.

Voilà donc qu'au Liebefeld j'ai pu affirmer que le lait sort de la mamelle en contenant déjà des microbes acidoprotéolytiques qui sont analogues à certains microbes du fromage, et auxquels on peut attribuer même la galactase de BABcock et RussELL.

(1) Ce principe a été confirmé par Barthel pour le Streptococcus lactis (Centr. f. Baleter, 1915, II. Abt. 44) et par VIrTANen pour le B. casei epsilon (Zeits. Phys. Chemie, 138, 1924). 
Encouragé par le résultat de mes nouvelles études sur l'extensibilité de ma conception, aussitôt retourné à Milan je les ai communiquées dans la même année 1901 dans une note à l'Istituto Lombardo di Scienze e Lettere, que M. Freudennreich a eu la bonté de traduire pour le Landwirtschaftliches Jahrbuch der Schweiz où elle a paru avec un an de retard, en même temps que dans la Revue Générale du Lait et dans le Centralblatt für Bakteriologie [6].

J'ai eu la satisfaction de constater qu'après mon passage au Liebefeld, Freudenreich et OrLA Jensen commencèrent à prendre en considération les coceus du fromage qu'ils appelèrent $M$. casei liquefaciens, et à admettre la possibilité, bien plus, la nécessité d'intervention de bactéries productrices d'enzymes protéolytiques, en modifiant

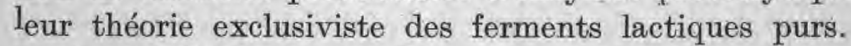

A vrai dire, pour quelque temps ils réfutèrent mes idées, en sou. tenant :

$1^{\circ}$ Que le $M$. Casei liquefaciens se sépare de mon coceus mammaire pour ses caractères morphologiques et culturaux;

$2^{\circ}$ Que, en tout cas, ces coccus n'ont pas d'importance dans la maturation des fromages parce que, quoique bien nombreux au début, ils disparaissent bientôt de la pâte.

Maís les recherches que, à partir de la dernière partie de l'année 1901 [7], j'ai poursuivies dans mon laboratoire, à l'Ecole supérieure d'agriculture de Milan, ont démontré :

$1^{\circ}$ Que les coccus mammaires aussi bien que les cocous du fromage ne sont pas d'un type unique, mais présentent une grande hétérogénéité de caractères morphologiques et physiologiques, qui amène à les diviser en plusieurs types et sous-types intermédiaires, dont j'en ai déerit deux principaux : l'un qui fond la gélatine ( $M$. acidoproteolyticus $I$ ), et l'autre qui ne fond pas la gélatine (M. acidoproteolyticus II) [8]; tous les types peuvent se rencontrer réunis dans la même souche, bien plus, dans la même colonie, présentant cette hétérogénéité et se prêtant à ces phénomènes de variation et même de mutation par divergence individuelle et par conditions de vie que j'ai démontrés récemment [9]. Partant, aujourd'hui je suis venu à l'idée de les embrasser tous sous le nom de Mammococcus ou Caseococcus selon l'habitat, en les rapprochant de l'Enterococcus et de mon Gastrococcus que j'ai isolé de la caillette de veau [10];

$2^{\circ}$ Que la disparition de ces coccus dans les fromages n'est pas si précoce; si on les recherche avec des méthodes appropriées, on peut les rencontrer encore après des mois [11]. Il faut surtout employer, non pas la méthode des plaques de Koch, mais celle des dilutions de Pasteur, et avoir soin de stériliser le lait de culture, non pas à l'autoclave, mais par tyndallisation à une température pas supérieure à $100^{\circ} \mathrm{C}$. et de tenir 
ces eultures à température basse. D'ailleurs, après la mort des cellules, leurs enzymes restent dans la pâte et elles sont susceptibles de poursuivre l'action peptonisante sur la caséine même à la température basse de maturation des fromages, ce qui justifierait la méthode américaine de maturation à froid (cold-curing) [12].

Je dirai en passant que l'action des diastases du Mammococcus est tout à fait aromatisante, caractéristique; pour en avoir une idée, il suffit de faire des plaques avec de l'agar additionné de lait trait aseptiquement de manière à ne pas tuer les mammocoques. En outre, puisque le développement du Mammococcus-caseococcus dans le fromage précède le développement des ferments lactiques purs, son action est extrêmement utile pour préparer à ces derniers la nourriture peptonisée dont ils ont besoin [13].

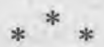

J'ai apporté une autre contribution à ma théorie:en 1904 [14], en déeouvrant dans le fromage Parmesan et aussi dans l'Emmenthal, un deuxième type de bactéries acidoprotéolytiques que j'ai décrit sous le nom de Bacillus acidificans presamigenes casei, le premier exemple d'un ferment lactique sporogène. Aussi ce type, dont en 1906 [15] j'ai démontré la provenance fourragère, a été accueilli pendant quelque temps avec un scepticisme décourageant; mais ensuite il a été retrouvé par moimême et par d'autres auteurs dans différents milieux lactés

Bien entendu, comme tous les microbes, il présente lui aussi des variétés, telle que le $B$. coagulans trouvé par SANDenrn dans la crème ; mais le type a son origine dans mon laboratoire de Milan, et non pas dans le laboratoire de Liebefeld, comme il est dit par erreur dans le Traitê de BEAU et Bourgatn (qui vraisemblablement croyaient qu'en 1904 je travaillais toujours au Liebefeld).

Je rappelerai encore, comme je l'ai démontré [16], que ce type de bacille est tout particulièrement hétérogène et susceptible de variation et même de mutation par divergences individuelles et par conditions de vie (température, aérobiose, etc.); chez lui aussi, j'ai constaté l'influence bienfaisante des températures basses sur la easéolyse.

$$
\text { *** }
$$

Des coccus et des bacilles acidoprotéolytiques similaires ont été désormais rencontrés dans toutes sortes de fromages par la plupart des auteurs. Je citerai : BurRI et ses élèves, pour l'Emmenthal; Bonkноut et DE VRIES, pour le fromage hollandais Edam; Russell et Hastings, Harding et Prucha, Alice Evans, Hart et Fuint, Hucker, pour le fromage américain Cheddar; Marcas et HuYge, pour le fromage belge; Barthel et Gerda Trom Peterson, pour le fromage suédoís; GrImmer, pour le fromage Tilsitt, KanTARDJEFF, 
pour le fromage bulgare; SADLER et KELLY, pour le fromage canadien Kingston; Gutsohy, pour le fromage serbe; VIRTANÉN, pour le fromage finlandais; Mazé et GuItTonneAU, pour le Gruyère; LAXA, pour les fromages bohémiens; DALLA ToRRE, pour le fromage italien Reggiano.

$$
*^{*} *
$$

Pour conclure, je dirai que l'ensemble de mes recherches m'autorise à présenter comme suit ma théorie acidoprotéolytique :

Dans la maturation des fromages jouent un rôle important les ferments mixtes acidoprotéolytiques, qui ont été découverts par moi (sans aucune collaboration) et qui forment des espèces hétérogènes susceptibles de mutations, tels que le Mammococcus qui est le ferment primitif du lait et le Bacillus acidificans presamigenes casei qui dérive des fourrages.

\section{BIBLIOGRAPHIE}

[1] M. Beau et Ch. Bourgatn, l'Industrie fromagère. Première Partie : la Science fromagère, Paris, J.-B. Baillière et fils, 1926.

[2] Atti dei Laboratori della Sanita Publica al Ministero Interni, Roma, 1892 ; Rivista d'Igiene e Sanita Pubblica, Roma, 1893, IV, p. 549. Mygienische Rundschau, 1893, III, p. 381; Giornale della R. Società Italiana d'Igiene, Milano, 1894, XVI, no 4.

[3] Bollettino Ufficiale del Ministero di Agricoltura, Roma, 1897.

[4] Annales de Micrographie, Paris, 1897, IX, no 11, p. 433.

[5] Rend. R. Ist. Lomb. Sc. e. Lett., 1901, 31, p. 1279.

[6] Landw. Jahrb. d. Schw., 1902, XVI, p. 22 ; Centralbl. f. Bakter. II Abt., 1902, 8, p. 137; Revue générale du Lait, 1902, I, p. 169.

[7] Rend. R. Accad. Lincei, 1902, XI, p. 159; Rend. R. Ist. Lomb. Sc. e. Lett., 1904, 37; Rend. R. Acad. Lincei, 1905, XIV, p. 396 ; Revue générale du Lait, 1904, III, n० 22; Milchwirts. Zentralblatt, 1905, H. 11.

[8] Rend. R. Accad. Lincei, 7 août 1910; Revue générale du Lait, 1910, VIII, $\mathrm{n}^{0} 15$; Milch. Zentralbl., 1911, H. 10; Zeits. $f$. Gärungsphysiologie, 1912, Bd. I.

[9] Rend. R. Accad. Lincei, 8 mai 1921 ; C. R. Acad. Sc., 30 mai 1921; Rend. Ist. Lomb. Sc. e Lett., 14 avril 1921 ; le Lait, 1922, II, $\mathrm{n}^{\circ} 1$; Milch. Forschungen, 1921, n० 10 ; Centr. J. Bakter., II, 1922, 55.

[10] C. R. Acad. Sc., 29 décembre 1924 et 16 novembre 1925; Rend. R. Accad. Lincei, janvier 1925; Revue Internat. de Renseignements agricoles, Rome, janvier-mars 1925; le Lait, 1926, VI, $\mathrm{n}^{\circ} 52$; Milch. Forschungen, 1926, 3, H. $2 / 3$; Centralb!. f. Bakter. II, 1926, 67.

[11] Voir [7].

[12] Rend. R. Accad. Linccei, 17 septembre 1911; Revue générale du Lait, 1912. IX, no 5 ; Centralbl. $f$. Bakter, II, 1912, 32. 
[13] Pendant la grande guerre, j'ai publié une série de travaux dans les $C$. $R$, de la R. Accad. Lincei et R. Ist. Lomb. Sc. e Lett,, années 1914-1920 dont j'ai donné un très court résumé dans : le Lait, 1921, I, n 2 ; Milch. Forschungen, janvier 1921; Centralbl. f. Bakter, II, 1921, 53, H. 13/14.

[14] Voir [7].

[15] Annuario Istituzione Agraria Ponti annexée à l'Ecole R. Sup. d'Agriculture de Milan, 1906, VI.

[16] Voir [9].

\section{TECHNIQUE DE LA STERILISATION DU LAIT}

\section{OBTENTION DE TEMPÉRATURES CONSTANTES ET D'UN PRODUIT DE CONDITION UNIFORME}

Par le Dr ROBIN

Directeur du Bureau Municipal d'Hygiène de Nantes chargé de la Laiterie Municipale.

Dans cette communication présentée en Mai 1926 au Congrès International de Laiterie, l'auteur faisait connaître qu'il avait été frappé des mêmes faits sur lesquels M. Pellet attire aujourd'hui l'attention, et il synthétisait dans un bref exposé contenant des indications pratiques sur la technique de la stérilisation, des observations auxquelles l'article de M. PrLlext apporte une intéressante contribution. (Le Lait, 1926 p. 593).

Les buts essentiels à atteindre dans la stérilisation sont l'uniformité de traitement à température fixe, la conservation de la couleur normale du lait, la réduction au maximum de la casse et de pertes de matière première.

L'auteur décrivait les différentes phases du travail sans négliger les détails de technique indispensable au succès dans une opération dont le principe n'est ignoré de personne :

$1^{0}$ Après introduction du lait dans l'autoclave et fermeture de celui-ci, on fait l'admission de vapeur, la vanne inférieure de vidange et le robinet d'air supérieur du couvercle restant partiellement ouverts. La vanne d'évacuation à l'air libre doit rester seule fermée.

On obtient ainsi un entraînement régulier de l'air intérieur et un échauffement du contenant progressif et égal à toutes les hauteurs de l'appareil où, le plus généralement, les flacons sont superposés par étages.

$2^{\circ}$ Admission lente de la vapeur de façon à obtenir au thermomètre extérieur $100^{\circ}$ en trente minutes.

Dès que la température de $90^{\circ}$ est obtenue, on étrangle progressivement la vanne de vidange inférieure, qui devra cependant rester ouverte pendant toute la durée de l'opération, ne laissant échapper qu'un filet de vapeur. 